\title{
Atresia biliar: una enfermedad grave
}

\author{
Biliary atresia: a severe illness
}

\author{
Dra. Margarita Ramonet ${ }^{a}$, Dra. Mirta Ciocca ${ }^{b}$ y Dr. Fernando Álvarez ${ }^{c}$
}

\begin{abstract}
RESUMEN
La atresia biliar es una grave enfermedad que se manifiesta en los recién nacidos, y se desconoce su causa. La inflamación y destrucción progresiva de los conductos biliares conducen a la aparición de ictericia, coluria y acolia entre la segunda y sexta semana de vida. Como existen múltiples causas de colestasis neonatal en esta etapa de la vida, es necesario realizar un diagnóstico y derivación precoz para ofrecer un tratamiento quirúrgico, con el fin de restablecer el flujo biliar. Alrededor del $80 \%$ de los pacientes normalizan la bilirrubina luego de la portoenterostomía (operación de Kasai), realizada antes de los 45 días de vida. Si la operación fracasa, el trasplante hepático surge como única alternativa. La atresia biliar debe diagnosticarse durante el primer mes de vida y ser considerada una urgencia quirúrgica. Palabras clave: atresia biliar, colestasis neonatal, recién nacido, bilirrubina directa, operación de Kasai.
\end{abstract}

\section{ABSTRACT}

Biliary atresia is a serious disease of unknown cause, affecting newborns. An inflammation and progressive destruction of the bile ducts lead to jaundice, dark urines, and acholia, between the second and sixth weeks of life. Neonatal cholestasis could be due to several different diseases, thus a diagnosis of biliary atresia and early derivation for surgical treatment are necessary to allow a restoration of the bile flow. Eighty percent of the children normalize serum bilirubin after the portoenterostomy (Kasai operation), if they are operated before their 45 days of life. When Kasai operation fails, a liver transplantation is the only possibility. Biliary atresia must be diagnosed before the first month of life and must be considered as a surgical emergency.

Key words: biliary atresia, neonatal cholestasis, newborns, direct bilirubin, Kasai operation.

http:/ /dx.doi.org/10.5546/aap.2014.542

\section{INTRODUCCIÓN}

La atresia biliar $(\mathrm{AB})$ es la causa más frecuente de ictericia obstructiva en los primeros tres meses de vida y es responsable del $40 \%$ al $50 \%$ de todos los trasplantes hepáticos en niños del mundo. Es el resultado final de un proceso inflamatorio fibroesclerosante y obstructivo que afecta a los ductus biliares intra- y extrahepáticos, que finaliza con la fibrosis y obliteración del tracto biliar y eventual desarrollo de cirrosis biliar con hipertensión portal y falla hepática, lo que provoca la muerte a los 2-3 años de vida si no media una intervención. ${ }^{1,2} \mathrm{La}$ hepatoportoenteroanastomosis temprana realizada en los dos primeros meses de vida ofrece la mejor oportunidad de supervivencia a largo plazo del paciente con hígado nativo.

Se estima que la incidencia mundial de la enfermedad oscila entre el 1 en 8000 y 18000 recién nacidos (RN) vivos; se presenta en todas las regiones del mundo, pero es más frecuente en el sexo femenino y en los

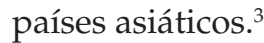

Existe firme consenso en la literatura pediátrica de que los esfuerzos dirigidos a la detección precoz de los casos de colestasis neonatal $(\mathrm{CN}), \mathrm{y}$ específicamente de la $A B$, no deben demorarse, ya que su pronóstico depende de un diagnóstico y tratamiento precoz. ${ }^{4,5}$

\section{PATOGENIA}

La AB es definida como la obliteración o ausencia del tracto biliar. Evidencias clínicas y experimentales sugieren que la expresión fenotípica de la enfermedad puede ser el resultado de una variedad de agentes etiológicos.

Actualmente, su caracterización incluye las siguientes variantes clínicas:

1) $A B$ asociada a otras malformaciones congénitas:

Esta variedad, a su vez, puede subdividirse en tres grupos:

- Síndrome BASM, que incluye malformaciones esplénicas (poliesplenia, asplenia, doble bazo). Además, se asocia a asimetría 
visceral (situs inversus y malrotación), malformaciones venosas (vena cava inferior ausente, vena porta preduodenal) y malformaciones cardíacas. Esta forma de $A B$ predomina en el sexo femenino $y$, en algunos casos, se vincula con antecedentes maternos de diabetes y tirotoxicosis. Se considera que la anormalidad del conducto biliar se produce al mismo tiempo que las otras anomalías mencionadas, durante el desarrollo embrionario, entre la $5^{\text {a }}$ y la $6^{\text {a }}$ semana de gestación. Esto ocurre antes de la formación del sistema ductal intrahepático (7-10 semanas).

- Otro grupo de pacientes con AB presentan hallazgos de otros síndromes, como el síndrome del ojo de gato (coloboma, atresia ano-rectal, etc.). Se incluye en las aneuploidías (alteración del número de cromosomas).

- Finalmente, algunos pacientes con $\mathrm{AB}$ tienen anomalías congénitas no sindrómicas, tales como la atresia esofágica, yeyunal, ano-rectal, etc. No existe para este grupo explicación genética convincente y podría obedecer al accionar de un agente causal durante el período embrionario.

2) AB quística:

En alrededor del 10\% de los casos, la AB incluye una formación quística y puede generar confusión con un quiste de colédoco. Esta formación se puede observar en una ecografía realizada durante el embarazo, a partir de las semanas 18-20 de gestación. Esta forma tiene una mejor evolución luego de la cirugía, posiblemente debido a la existencia de una favorable continuidad luminal con los conductos intrahepáticos.

3) AB aislada:

Es el grupo más numeroso, con igual distribución por género. En relación con la etiología, se mencionan dos hipótesis:

- El tiempo de comienzo sería más tarde que en los grupos sindromáticos, ya que no se asocian al compromiso de otros órganos o sistemas.

- La hipótesis actual para explicar este tipo de $A B$ es que estos pacientes desarrollan un tracto biliar completo y la obliteración sería un fenómeno secundario que ocurriría en el período perinatal. La responsabilidad de algunos virus hepatotrópicos en el desarrollo de este tipo de $A B$ ha sido un tema controvertido desde la década de los 80. En un estudio reciente, la búsqueda de posibles virus hepatotrópicos mostró que un ARN/ADN viral fue detectado en el hígado en menos del $50 \%$ de los casos (reovirus:
$33 \%$; citomegalovirus: $11 \%$; adenovirus: $1 \%$; y enterovirus: $1,5 \%$ ). Lo que no permite establecer esta publicación es si estos virus son transeúntes inocentes o responsables del mecanismo colangiodestructivo. ${ }^{6-8}$

La hipótesis de una infección viral responsable de esta enfermedad se basa en la posibilidad de que, una vez eliminado el virus, una respuesta autoinmune provocada por la infección perpetuaría el estado inflamatorio. A nivel experimental, los ratones Balb/c (albinos) son las cepas más susceptibles y el rotavirus RRV (rotavirus rhesus grupo A) administrado por vía intraperitoneal dentro de las primeras 24 horas de vida es el único virus capaz de inducir cambios necroinflamatorios biliares. El virus resulta indetectable a la segunda semana postexposición, y persiste la respuesta inmune celular. La reducción de células $\mathrm{T}$ reguladoras (controladoras de la inflamación), en número y función durante los primeros días de vida, facilitaría el desarrollo del compromiso biliar. Otro hecho de observación, demostrado en el modelo animal, está vinculado con la vacunación materna contra el RRV, la cual generaría la producción de anticuerpos IgG neutralizantes, que participarían en la eliminación del virus. ${ }^{9-14}$

\section{CLÍNICA}

En los casos de ictericia que se prolonga más allá de las dos semanas de vida, es imprescindible realizar la determinación de la bilirrubina directa (BD) sin demoras. ${ }^{1-3}$

La presentación clínica es habitualmente en un recién nacido de término, con peso adecuado, aspecto normal, que comienza con ictericia progresiva, acolia entre las 2 y las 6 semanas de vida. La coluria y la decoloración de las heces son elementos importantes en el examen clínico. La hepatomegalia se encuentra siempre presente, de consistencia aumentada y, en ocasiones, acompañada de esplenomegalia.

Los hallazgos de laboratorio incluyen el incremento de la BD, elevación moderada de las transaminasas, gamaglutamiltranspeptidasa elevada, fosfatasa alcalina usualmente elevada, albúmina y glucemia normales; los triglicéridos y el colesterol están habitualmente normales, y la función hepática, normal.

\section{DIAGNÓSTICO}

El diagnóstico debe ser considerado en primer lugar frente a un lactante de un mes de edad, ictérico, con heces acólicas y hepatomegalia. 
El proceso del diagnóstico de la $\mathrm{AB}$ no debe demorarse y continúa siendo un desafío para el pediatra.

La ecografía abdominal se debe realizar con 4 horas de ayuno para poder visualizar la forma y el tamaño de la vesícula o su ausencia. El área triangular o signo del cordón fibroso, que corresponde a un área ecogénica del portahepatis, es posiblemente un hallazgo específico de $\mathrm{AB}$. Además, permite identificar malformaciones vasculares asociadas al síndrome de poliesplenia (AB forma fetal), como la vena porta preduodenal, agenesia de la vena cava, y otras patologías biliares, como el quiste del colédoco, y la litiasis., ${ }^{1,15}$

La biopsia hepática percutánea es un elemento de extrema importancia, con un alto grado de especificidad, de hasta un $95 \%$, para poder arribar al diagnóstico, con una muestra apropiada (más de 10 espacios porta) y un patólogo con experiencia en patología hepática pediátrica.

Los hallazgos histológicos más frecuentes son la proliferación ductular, estasis hepatocitaria de los pequeños conductos biliares, fibrosis $\mathrm{y}$, a veces, transformación gigantocelular. La laparotomía/laparoscopía exploradora con colangiografía debe ser efectuada precozmente ante la sospecha clínica. ${ }^{1,15,16}$

Una serie de parámetros clínicos permiten orientar el diagnóstico diferencial de la AB: la observación diaria del color de las deposiciones durante 10 días consecutivos, el peso de nacimiento, la edad de comienzo de la acolia y las características de la hepatomegalia. Si el peso al nacer era normal, el comienzo de la acolia, precoz y constante, y la hepatomegalia era firme, la posibilidad de que fuera una AB fue del $82 \% .{ }^{17,18}$

En otro estudio, la determinación de la bilirrubina directa, en todos los neonatos que continuaban ictéricos luego de los 14 días de vida, permitió derivar a aquellos con niveles anormales de bilirrubina directa a centros de mayor complejidad para su diagnóstico etiológico y tratamiento. ${ }^{19,20}$
Matsui y Dodoriki (1995), en Japón, con un sistema de tarjetas colorimétricas, realizaron un tamizaje en una población de $17641 \mathrm{RN}$ en el control de salud al mes de edad e identificaron 2 casos de $\mathrm{AB}$ de 3 que presentaban deposiciones hipo-/acólicas, por lo que lograron realizar la operación de Kasai antes de los 60 días de vida. ${ }^{21}$

El tamizaje universal fue establecido en Taiwán utilizando un método similar. La sensibilidad para la detección de la $\mathrm{AB}$ con el uso de tarjetas con el color de la materia fecal fue de $72,5 \%$ en 2004 y de $97,1 \%$ en 2005 . La detección precoz aumentó el número de pacientes operados antes de los 60 días de vida. ${ }^{22}$

En nuestro país, se realizó un estudio en el Hospital Nacional Prof. A. Posadas (período 19992002).

Fue un estudio piloto, prospectivo, observacional, en el que se usó el método de tamizaje de las heces con tarjetas colorimétricas en todos los RN que nacieran en el Hospital y fueran atendidos en el control del primer mes. De un total de 12484 niños, 4239 (33,9\%) concurrieron a la visita del primer mes con la tarjeta colorimétrica. Se identificaron 18 con deposiciones hipo-/acólicas, de los cuales solo 4 presentaron enfermedad colestática. Los diagnósticos definitivos fueron síndrome de Alagille, hepatitis luética, colestasis neonatal transitoria y litiasis biliar. Si bien no se identificó ningún caso de $A B$, la prueba de tamizaje demostró ser de utilidad para la detección de otras causas de CN. Sobre la base de esta experiencia, se decidió la implementación del tamizaje con las tarjetas colorimétricas, como una práctica de rutina en nuestro Hospital. Hasta el presente, esta estrategia permitió la detección de niños con colestasis neonatal, que incluye dos casos de $\mathrm{AB}$ a los 38 y 42 días de vida, en los cuales fue posible realizar precozmente la intervención de Kasai. ${ }^{23,24}$ (Tabla 1).

La colangiopancreatografía retrógada endoscópica no es tan utilizada en niños

TABla 1. Diferentes experiencias con la utilización de las tarjetas colorimétricas

\begin{tabular}{lcccc}
\hline Autor/año/país & Población & Casos de AB & Otras CN & Kasai (días) \\
\hline Matsui/95/Japón & 17641 & $2 / 3$ & - & 35 y 69 \\
Chen/06/Taiwán & 119973 & $29 / 30$ & 8 HNI/3 otras & $29<90$ \\
Hsiao/08/Taiwán & 422264 & 75 & - & $84 \%$ (<60 días) \\
Ramonet/13/Argentina & 12484 & $*$ & 4 : SAlag, ${ }^{* * H N I, ~ l i t i a s i s, ~ H L u ́ e s ~}$ & 38 y 42 \\
\hline
\end{tabular}

CN: colestasis neonatal. ${ }^{*}$ Después del estudio piloto. ${ }^{* *}$ HNI: hepatitis neonatal inespecífica. 
como en adultos y se realiza en pocos centros especializados en el mundo. ${ }^{25}$

Un estudio de cohorte retrospectivo para investigar la relación entre el color de la piel y la edad de derivación de los pacientes con $\mathrm{AB}$ mostró que los pacientes no blancos fueron derivados más tardíamente que el grupo control (blancos), con una diferencia significativa ( $\mathrm{p}<$ $0,02) \cdot{ }^{26,27}$

Las recomendaciones recientes de las Guías de Evaluación de la Colestasis de la Asociación Norteamericana de Gastroenterología, Hepatología y Nutrición (North American Society for Pediatric Gastroenterology, Hepatology and Nutrition; NASPGHAN, por sus siglas en inglés) son las siguientes: $:^{19,20}$

- Las Guías proveen recomendaciones para el manejo en el $1^{\mathrm{er}}$ nivel de atención, indicaciones de consulta con el especialista y manejo del pediatra hepatólogo.

- La meta continúa siendo la detección precoz y el diagnóstico eficiente.

- No hay un test de tamizaje que pueda predecir qué lactante va a desarrollar colestasis.

- La recomendación es que cualquier recién nacido que presente ictericia a las 2 semanas de vida debe ser evaluado con la determinación de BD.

\section{TRATAMIENTO: CIRUGÍA, MÉDICO}

Cuando la enfermedad es sospechada, la intervención permite realizar una colangiografía intraoperatoria para confirmar el diagnóstico, seguido en el caso de $\mathrm{AB}$ de una portoenterostomía u operación de Kasai, única posibilidad terapéutica.

La portoenterostomía descrita en 1959 es un tratamiento destinado a restablecer el flujo biliar. Durante la intervención, los conductos biliares microscópicos situados en la porta hepatis son expuestos y drenados a través de la anastomosis de un asa de yeyuno en Y de Roux a los bordes fibrosos de esta para restablecer el flujo biliar. Es muy importante realizar la intervención lo antes posible luego del nacimiento para tener mayores posibilidades de un resultado exitoso. Por ello, es necesario enfatizar la derivación temprana a un centro de referencia de todo paciente con una colestasis durante el período neonatal. En aquellos casos en los que la portoenterostomía no logra restablecer un flujo biliar suficiente, el paciente deberá ser evaluado para un trasplante hepático.

Cuando la intervención es exitosa, se reducen los niveles de bilirrubina y puede llegar a normalizarse. Este resultado permite una prolongada supervivencia del paciente con $A B$, con su hígado nativo.

Aunque la mayoría de los niños con $A B$ requerirán un trasplante, la portoenterostomía permite, en muchos casos, retrasar su indicación hacia la segunda infancia, la adolescencia o la adultez. Esta realidad resulta ventajosa ya que, con el crecimiento del niño, es más fácil encontrar un donante adecuado, se reducen las dificultades técnicas y, con ellas, las complicaciones quirúrgicas del trasplante.

La historia natural del paciente con $\mathrm{AB}$ no operado o con una falla de la intervención se asocia a colestasis crónica, fibrosis hepática, cirrosis e insuficiencia hepática.

Se han implementado algunos tratamientos médicos luego de la intervención, destinados a estimular el flujo biliar, prevenir la colangitis y reducir la inflamación hepática y fibrosis progresiva asociada con la enfermedad. Algunos centros indican profilaxis con antibióticos orales para disminuir los episodios de colangitis y existen escasos datos publicados que respalden las ventajas de su administración. Otros programas indican corticosteroides durante un período de tiempo variable, debido a sus acciones antiinflamatorias, inmunomoduladoras y estimulante del flujo biliar (dependiente de sales biliares). Un estudio controlado no encontró beneficio en la supervivencia del hígado nativo; únicamente se observó que los pacientes que recibieron tratamiento presentaron menor nivel de bilirrubina sérica en los seis meses posteriores.

El ácido ursodesoxicólico oral es también comúnmente utilizado, en dosis variables, como estimulante del flujo biliar y por su posible efecto protector hepático. ${ }^{28-30}$

\section{EVOLUCIÓN Y SEGUIMIENTO, PREVENCIÓN DE LAS COMPLICACIONES}

En el seguimiento del paciente con AB luego de la portoenterostomía, más de la mitad de los pacientes normalizan la bilirrubina dentro de los seis meses luego de la cirugía, y la supervivencia actuarial a los 5 años con el hígado nativo es del $30 \%$ al $60 \%$. Aproximadamente el $20 \%$ de los pacientes sometidos a la intervención sobreviven más allá de los 18 años con su hígado nativo. El nivel de bilirrubina sérico luego de la cirugía es el predictor evolutivo más importante. Su normalización nos anticipa una evolución favorable en el largo plazo. Otros factores que 
afectan la evolución del hígado nativo son la edad al momento de la cirugía, la extensión del daño hepático (fibrosis) y la experiencia del equipo quirúrgico (Tablas 2 y 3 ).

Las complicaciones más frecuentes, luego de una intervención de Kasai exitosa, incluyen la colangitis ascendente y la hipertensión portal. Ambas pueden ocurrir con un nivel de bilirrubina normal.

Los episodios de colangitis habitualmente se observan en las semanas siguientes a la portoenterostomía. Clínicamente, se caracterizan por dolor abdominal, fiebre, parámetros de inflamación elevados y aumento variable de niveles de bilirrubina, GGT y aminotransferasas. Un esfuerzo particular debe realizarse para lograr identificar la bacteria o el hongo responsable de la infección, para adecuar el tratamiento antibiótico por vía intravenosa y que se prolongará por tres semanas. Los episodios de colangitis recurrentes pueden estar asociados con dilataciones quísticas intrahepáticas, las cuales son evidenciadas por ecografía y constituyen un signo de mal pronóstico.

El síndrome de hipertensión portal puede manifestarse con rapidez, en especial en los casos de fracaso de la portoenterostomía. Clínicamente, se va a exteriorizar con esplenomegalia y várices esofágicas. Una proporción significativa de estos pacientes van a presentar hemorragia variceal. La presencia de ascitis se observa en casos de hemorragia digestiva, desnutrición o insuficiencia hepática, cuando a la hipertensión portal se asocia una disminución de la albúmina sérica. Algunos centros realizan control endoscópico anual de las várices esofágicas e indican escleroterapia o ligadura profiláctica ante la presencia de estas..$^{29-32}$

\section{TRASPLANTE HEPÁTICO}

La $\mathrm{AB}$ es la indicación de trasplante hepático pediátrico más frecuente. La mayoría de los pacientes que no han obtenido la restauración del flujo biliar serán trasplantados durante el primer año de vida. Otros parámetros clínicos que nos van a orientar hacia su indicación son los siguientes: ascitis refractaria, peritonitis bacteriana, colestasis progresiva, deterioro de la función hepática o presencia de un síndrome hepatopulmonar. La supervivencia anual del paciente con $\mathrm{AB}$ trasplantado hepático progresó desde $75 \%$ en los 80 a más del $90 \%$ actualmente. En el seguimiento del paciente trasplantado, la supervivencia documentada fue del $81 \%, 80 \%$ y $77 \%$ a los 5, 10 y 20 años, respectivamente. La supervivencia total a los 5 años, en otras series recientes, es del 90\%. La presencia de anomalías vasculares complejas entre los receptores con BASM está asociada con más complicaciones y una menor supervivencia luego del trasplante. ${ }^{31-35}$

\section{CONCLUSIONES}

Cuando estamos frente a un RN ictérico, debemos realizar lo siguiente:

TABla 2. Supervivencia con el hígado nativo - Francia, 1986-2009

\begin{tabular}{lccc}
\hline Edad al Kasai & N pacientes & A los 10 años & A los 20 años \\
\hline 60 días & 459 & $38,8 \%$ & $29,4 \%$ \\
$61-90$ días & 316 & $30 \%$ & $26,4 \%$ \\
$91-120$ días & 81 & $24,5 \%$ & $16,6 \%$ \\
$121-150$ días & 22 & $5,2 \%$ & 0 \\
$>$ 150 días & 8 & 0 & 0 \\
\hline
\end{tabular}

TABLA 3. Supervivencia con el hígado nativo - Japón, 1953-2009

\begin{tabular}{lccc}
\hline Edad al Kasai & N pacientes & A los 10 años & A los 20 años \\
\hline$\leq 60$ días & 67 & $55 \%$ & $43 \%$ \\
$61-90$ días & 114 & $45 \%$ & $33 \%$ \\
$91-120$ días & 38 & $28 \%$ & $25 \%$ \\
$121-150$ días & 15 & $7 \%$ & $7 \%$ \\
$>150$ días & 8 & 0 & 0 \\
\hline
\end{tabular}


1. Confirmar el diagnóstico de colestasis.

2. Pensar en $\mathrm{AB}$, una enfermedad no tan frecuente.

3. El diagnóstico debe ser rápido.

4. Es una urgencia quirúrgica.

\section{REFERENCIAS}

1. De Bruyne R, Van Biervliet S, Vande Velde S, Van Winckel M. Clinical practice: neonatal cholestasis. Eur J Pediatr 2011;170(3):279-84.

2. Sokol RJ, MackC,Narkewicz MR, Karrer FM. Pathogenesis and outcome biliary atresia: current concepts. J Pediatr Gastroenterol Nutr 2003;37(1):4-21.

3. Sokol RJ, Shepherd RW, Superina R, Bezerra JA, et al. Screening and outcomes in biliary atresia: summary of a National Institutes of Health workshop. Hepatology 2007;46(2):566-81.

4. Suchy FJ. Approach to the infant with cholestasis. En:Suchy FJ, Sokol RJ, Balistreri WF, eds. Liver Diseases in children. $2^{\text {nd }}$ ed. Philadelphia: Lippincott Williams \& Wilkins; 2001. Págs.187-94.

5. Schwarz KB, Haber BH, Rosenthal P, Mack CL, et al. Extrahepatic anomalies in infants with biliary atresia: results of a large prospective North American multicenter study. Hepatology 2013;58(5):1724-31.

6. Petersen C, Davenport M. Aetiology of biliary atresia: what is actually known? Orphanet J Rare Dis 2013;8:128.

7. Makin E, Quaglia A, Kvist N, Petersen BL, et al. Congenital biliary atresia: liver injury begins at birth. J Pediatr Surg 2009;44(3):630-3.

8. Davenport M. Biliary atresia: clinical aspects. Semin Pediatr Surg 2012;21(3):175-84.

9. Rauschenfels S, Krassmann M, Al-Masri AN, Verhagen W, et al. Incidence of hepatotropic viruses in biliary atresia. Eur J Pediatr 2009;168(4):469-76.

10. Mack CL, Sokol RJ. Unraveling the pathogenesis and etiology of biliary atresia. Pediatr Res 2005;57(5Pt2):87R-94R.

11. Beland K, LapierreP, Djilali-Saiah I, Alvarez F. Liver restores immune homeostasis after local inflammation despite the presence of autoreactive T cells. PLoS One 2012;7(10):e48192.

12. Mack CL, Tucker RM, Lu BR, Sokol RJ, et al. Cellular and humoral autoimmunity directed at bile duct epithelia in murine biliary atresia. Hepatology 2006;44(5):1231-9.

13. Tucker RM, Feldman AG, Fenner EK, Mack CL. Regulatory T cells inhibit Th1 cell-mediated bile duct injury in murine biliary atresia. J Hepatol 2013;59(4):790-6.

14. Alvarez F. Is biliary atresia an immune mediated disease? J Hepatol 2013;59(4):648-50.

15. Roberts E. The jaundiced baby. En: Kelly D, ed. Diseases of the Liver and Biliary System in Children. $3^{\text {rd }}$ ed. Hong Kong: Wiley-Blackwell; 2008. Págs.57-105.

16. Bellomo-Brandao MA, Escanhoela CA, Meirelles LR, Porta $\mathrm{G}$, et al. Analysis of the histologic features in the differential diagnosis of intrahepatic neonatal cholestasis. World $J$ Gastroenterol 2009;15(4):478-83.

17. Eliot N, Odievre M, Hadchouel M, Hill C, et al. Analyse statistique des données cliniques, biologiques et histologiques dans 288 observations de cholestase neonatale. Arch Fr Pediatr 1977;34(7 Suppl):CCXIII-CCXX.

18. Alagille D. Cholestasis in the first three months of life. Prog Liver Dis 1979;6:471-85.

19. Mowat AP, Davidson LL, Dick MC. Earlier identification of biliary atresia and hepatobiliary disease: selective screening in the third week of life. Arch Dis Child 1995;72(1):90-2.

20. Neimark E, Leteiko NS. Early detection of biliary atresia raises questions about etiology and screening. Pediatrics 2011;128(6):e1598-9.

21. Matsui A, Dodoriki M. Screening for biliary atresia. Lancet 1995;345(8958):1181.

22. Hsiao $\mathrm{CH}$, Chang MH, Chen HL, Lee HC, et al. Universal screening for biliary atresia using an infant stool color card in Taiwan. Hepatology 2008;47(4):1233-40.

23. Ramonet MD, Gómez S, Morise S, Parga L, et al. Detección precoz de la colestasis neonatal en las heces por el método de tamizaje con tarjetas colorimétricas. Arch Argent Pediatr 2013;111(2):135-9.

24. Shteyer E, Wengrower D, Benuri-Silbiger I, Gozal D, et al. Endoscopic retrograde cholangiopancreatography in neonates cholestasis. J Pediatr Gastroenterol Nutr 2012;55(2):142-5.

25. Tseng JJ, Lai MS, Lin MC, Fu YC. Stool color card screening for biliary atresia. Pediatrics 2011;128(5):e1209-15.

26. Martin LR, Davenport M, Dhawan A. Skin colour: a barrier to early referral of infants with biliary atresia in UK. Arch Dis Child 2012;97(12):1102-3.

27. Benchimol EI, Walsh CM, Ling SC. Early diagnosis of neonatal cholestatic jaundice: test at 2 weeks. Can Fam Physician 2009;55(12):1184-92.

28. Gallo A, Esquivel CO. Current options for management of biliary atresia. Pediatr Transplant 2013;17(2):95-8.

29. Willot S, Uhlen S, Michaud L, Briand G, et al. Effect of ursodeoxycholic acid on liver function in children after successful surgery for biliary atresia. Pediatrics 2008;122(6):e1236-41.

30. Shneider BL, Magee JC, Bezerra JA, Haber B, et al. Efficacy of fat-soluble vitamin supplementation in infants with biliary atresia. Pediatrics 2012;130(3):e607-14.

31. Chardot C, Buet C, Serinet MO, Golmard JL, etal.Improving outcomes of biliary atresia: French national series 19862009. J Hepatol 2013;58(6):1209-17.

32. Lykavieris P, Chardot C, Sokhn M, Gauthier F, et al. Outcome in adulthood of biliary atresia: a study of 63 patients who survived for over 20 years with their native liver. Hepatology 2005;41(2):366-71.

33. Nio M, Sasaki H, Wada M, Kazama T, et al. Impact of age at Kasai operation on sort- and long-term outcomes of type III biliary atresia at a single institution. J Pediatr Surg 2010;45(12):2361-3.

34. Serinet $\mathrm{MO}$, Broué $\mathrm{P}$, Jacquemin $\mathrm{E}$, Lachaux $\mathrm{A}$, et al. Management of patients with biliary atresia in France: results of a decentralized policy 1986-2002. Hepatology 2006;44(1):75-84.

35. Pakarinen MP, Rintala RJ. Surgery of biliary atresia. Scand J Surg 2011;100(1):49-53. 